\title{
Predição do Desempenho Aeróbio na Canoagem a Partir da Aplicação de Diferentes Modelos Matemáticos de Velocidade Crítica
}

\author{
Aerobic Performance Prediction in Canoeing from the Application of \\ Different Mathematical Models of Critical Velocity
}

Fábio Yuzo Nakamura ${ }^{1,2}$

Thiago Oliveira Borges 1,2

Fernando Roberto De-Oliveira ${ }^{3}$ Lúcio Flávio Soares-Caldeira Rômulo Cássio de Moraes Bertuzzi ${ }^{1,4,5}$

Karin Ayumi Matsushigue ${ }^{1,6}$

1. Grupo de Estudo das Adaptações Fisiológicas ao Treinamento (GEAFIT). Centro de Educação Física e Esporte. Universidade Estadual de Londrina - Londrina/PR.

2. Grupo de Estudo e Pesquisa em Metabolismo, Nutrição e Exercício. Centro de Educação Física e Esporte. Universidade Estadual de Londrina - Londrina/PR.

3. Núcleo de Estudos do Movimento Humano Universidade Federal de Lavras/MG.

4. Laboratório de Desempenho Esportivo. Escola de Educação Física e Esporte. Universidade de São

Paulo - São Paulo/SP.

5. Centro de Educação Física. Universidade Presbiteriana Mackenzie - Barueri/SP. 6. Pontifícia Universidade Católica do Paraná. Centro de Teologia e Ciências Humanas. Departamento de Educação Física - Curitiba/PR.

\section{Endereço para correspondência: Fábio Yuzo Nakamura Grupo de Estudo das Adaptações Fisiológicas ao Treinamento Centro de Educação Física e Esporte Universidade Estadual de Londrina Rod. Celso Garcia Cid, km 380, \\ Campus Universitário \\ CEP 86051-990 - Londrina, PR - Brasil \\ E-mail: \\ fabioy_nakamura@yahoo.com.br}

Submetido em 29/07/2005

Versão final recebida em 20/04/2008 Aceito em 21/04/2008

\section{RESUMO}

O modelo de velocidade crítica com dois parâmetros vem sendo utilizado para a determinação do desempenho aeróbio e anaeróbio. No entanto, o modelo com três parâmetros prevê um parâmetro adicional correspondente à velocidade instantânea máxima $\left(V_{\text {máx }}\right)$ ao modelo original, tendo impacto sobre a estimativa dos outros dois parâmetros. Sendo assim, o objetivo deste trabalho foi verificar e comparar a relação da velocidade crítica (VCrit), fornecida pelos modelos com dois (VCrit- $2_{\text {parâmetros }}$ ) e três (VCrit- $\left.3_{\text {parâmetros }}\right)$ parâmetros, com a velocidade média mantida em esforço máximo de $6.000 \mathrm{~m}\left(\mathrm{~V}_{6000 \mathrm{~m}}\right)$ na canoagem. Nove canoístas (17,4 \pm 2,4 anos) competitivos do sexo masculino realizaram, em dias diferentes, testes máximos de 200, 500, 1.000, 1.800 e $6.000 \mathrm{~m}$. A VCrit foi calculada para todos os sujeitos, a partir das distâncias de 200 a $1.800 \mathrm{~m}$, utilizando-se as equações hiperbólicas com dois e três parâmetros. As durações dos percursos ficaram no intervalo entre 46,1 \pm 2,5s e 513,9 \pm 25,6s. Para comparação entre as velocidades (VCrit- $2_{\text {parâmetrosı }}$ VCrit- $3_{\text {parâmetros }}$ e $V_{6000 m}$ ) foi utilizada ANOVA one way para medidas repetidas, seguida pelo teste post hoc de Newman-Keuls. A associação entre as variáveis foi feita a partir de correlação de Pearson. Para análise de concordância foi aplicada a plotagem de Bland-Altman entre os valores de VCrit- $2_{\text {parâmetros' }}$ VCrit- $3_{\text {parâmetros }}$ e $V_{6000 m}$. Em média, 0 tiro de $6.000 \mathrm{~m}$ teve duração de $29,9 \pm 1,0 \mathrm{~min}$. Houve diferença significante da VCrit-3 parâmetros $(2,93 \pm 0,36 \mathrm{~m} / \mathrm{s})$ em relação à VCrit-2 $2_{\text {parâmetros }}\left(3,31 \pm 0,22 \mathrm{~m} \cdot \mathrm{s}^{-1}\right)$ e $V_{6000 \mathrm{~m}}\left(3,35 \pm 0,11 \mathrm{~m} \cdot \mathrm{s}^{-1}\right)$. VCrit-2 parâmetros $\mathrm{V}_{6000 \mathrm{~m}}$ não diferiram entre si. A correlação entre VCrit- $2_{\text {parâmetros }}$ e $V_{6000 m}$ foi de $0,72(p=0,03)$, enquanto que a entre VCrit- $3_{\text {parâmetros }}$ e $V_{6000 m}$ não foi significante. Os resultados deste estudo sugerem que o modelo de velocidade crítica de dois parâmetros fornece valor de VCrit mais adequado para a avaliação aeróbia de canoístas. A VCrit- 3 parâmetros Subestima a velocidade que pode ser mantida por aproximadamente $30 \mathrm{~min}$, com baixa capacidade preditiva de desempenho aeróbio. Assim, foram obtidas evidências de validade do modelo original de velocidade crítica de dois parâmetros proposto por Monod e Scherrer.

Palavras-chave: velocidade crítica, dois parâmetros, três parâmetros, canoagem.

\section{ABSTRACT}

The 2-parameter critical velocity model has been used for determination of aerobic and anaerobic performance. Nevertheless, the 3-parameter model previews an additional parameter corresponding to the maximal instantaneous velocity $\left(V_{\max }\right)$ to the original model, having an impact on the estimation of the other two parameters. Thus, the aim of this work was to verify and compare the relationship of the critical velocity $\left(C_{\text {crit }}\right)$, provided by the 2-parameter $\left(C_{\text {crit-2parameters }}\right)$ and 3-parameter $\left(V_{\text {crit-3parameters }}\right)$ models, with the mean velocity kept in a maximal effort of $6,000 \mathrm{~m}\left(\mathrm{~V}_{6000 \mathrm{~m}}\right)$ in canoeing. Nine competitive, male canoers $(17.4 \pm 2.4$ years) performed at different days, maximal tests of $200,500,1,000,1,800$ and $6,000 \mathrm{~m}$. The $V_{\text {crit }}$ was calculated for all subjects, from the 200 to 1,800 distances, using the hyperbolic equations with two and three parameters. The distance durations were in the interval between $46.1 \pm 3.5 \mathrm{~s}$ to $513.9 \pm 25.6 \mathrm{~s}$. One-way ANOVA for repeated measures was used for comparison between velocities $\left(V_{\text {crit-2parameters }} V_{\text {crit-3parameters }}\right.$ and $\left.V_{6000 m}\right)$, followed by the Newman-Keuls post hoc. The association between variables was performed with the Pearson correlation. The Bland-Altman Plot among the $V_{\text {crit-2parameters }} V_{\text {crit-3parameters }}$ and $V_{6000 m}$ was applied for concordance analysis. The $6,000 \mathrm{~m}$ sprint had an average of $29.9 \pm 1.0$ min duration. There was significant difference of the $V_{\text {crit-3parameters }}$ $(2.93 \pm 0.36 \mathrm{~m} / \mathrm{s})$ in comparison with the $V_{\text {crit-2parameters }}\left(3.31 \pm 0.22 \mathrm{~m} . \mathrm{s}^{-1}\right)$ and $V_{6000 \mathrm{~m}}\left(3.35 \pm 0.11 \mathrm{~m} \cdot \mathrm{s}^{-1}\right) . \mathrm{V}_{\text {crit- }}$ 2parameters and $V_{6000 m}$ did not differ between each other. The correlation between $V_{\text {crit-2parameters }}$ and $V_{6000 m}$ was of 0.72 ( $p=0.03$ ), while the correlation between $V_{\text {crit-3parameters }}$ and $V_{6000 m}$ was not significant. The outcomes of this study suggest that the 2-parameter critical velocity model provides $V_{\text {crit }}$ values more suitable for the aerobic assessment of canoers. The $V_{\text {crit-3parameters }}$ underestimates the velocity which can be kept for approximately 30 min, with low aerobic performance predictive capacity. Thus, evidence on the validity of the 2-parameter original model critical velocity proposed by Monod and Scherrer was obtained.

Keywords: critical velocity, two parameters, three parameters, canoeing. 


\section{INTRODUÇÃO}

O modelo de potência crítica tem sua origem em 1965, quando Monod e Scherrer ${ }^{(1)}$ estabeleceram uma relação entre a carga imposta em exercícios monoarticulares e seus respectivos tempos até a exaustão para determinação de dois parâmetros bioenergéticos, a partir de uma função não-linear. Assumindo esse modelo, a interpretação do desempenho é feita com base na estimativa desses dois parâmetros, um aeróbio (potência crítica - PCrit) e outro anaeróbio (capacidade de trabalho anaeróbio - CTAnaer), os quais estão associados à exaustão em exercício severo(2)

Tradicionalmente, a PCrit e a CTAnaer têm sido estimadas a partir de uma hipérbole, com duas assíntotas, uma no eixo da potência e outra no eixo do tempo ${ }^{(1,3)}$. A assíntota no eixo da potência tem sido interpretada como intensidade teórica em que o esforço poderia ser mantido indefinidamente, a qual tem sido denominada PCrit. Na prática, essa intensidade não pode ser mantida indefinidamente, mas sim por cerca de 30 minutos $^{(4)}$, sendo associada a um máximo estado estável de consumo de oxigênio $\left(\mathrm{VO}_{2}\right)$ e das concentrações sangüíneas de lactato ${ }^{(2,5-7)}$. Dessa forma, abaixo dessa intensidade, a fadiga não deve ocorrer por acúmulo de lactato. Já acima dela, é provável que a utilização da CTAnaer associe-se diretamente à taxa de acúmulo dessa substância nos músculos e no sangue, causando a exaustão. Além disso, a CTAnaer tem sido relacionada ao máximo déficit de acúmulo de oxigênio ${ }^{(8)}$. Contudo, a assíntota positiva no eixo do tempo não teria explicação fisiológica razoável, pois assume que a estrutura musculoesquelética seria capaz de realizar uma ação muscular instantânea com potência tendendo a infinito.

Tentando corrigir essa falha matemática no modelo de dois parâmetros, Morton ${ }^{(9)}$, em 1996, propôs um modelo que forçava a hipérbole a necessariamente cruzar o eixo da potência (intercepto-x), acrescentando, assim, um parâmetro chamado potência máxima $\left(P_{\text {máx }}\right)$, que seria a potência instantânea em tempo tendendo a zero.

A inserção do terceiro parâmetro e a aplicação desse modelo a dados experimentais têm implicado a obtenção sistemática de valores inferiores de PCrit em relação ao modelo de dois parâmetros ${ }^{(9-13)}$ e valores superiores de CTAnaer ${ }^{(9,13)}$ que, segundo Morton ${ }^{(9)}$, o que não seria completamente utilizado durante o exercício acima da PCrit. Em outras palavras, mesmo ao final de um exercício severo exaustivo, alguma porção da CTAnaer seria preservada nos músculos ativos, na forma dos estoques de glicogênio, principalmente ${ }^{(10)}$. Segundo Vandewalle et al. ${ }^{(14)}$, a não consideração desse fato seria um dos fatores limitantes dos pressupostos do modelo tradicional de dois parâmetros.

O modelo de potência crítica de dois parâmetros vem sendo aplicado no meio esportivo, sobretudo em esportes cíclicos como corrida e natação, com a denominação ajustada para velocidade crítica $(\mathrm{VCrit})^{(3,15,16)}$.

Ainda assim, são poucas as evidências a favor da validade do modelo de potência crítica ou de velocidade crítica de três parâmetros ${ }^{(10)}$. Além disso, elas têm falhado em estabelecer um significado fisiológico bem definido para os parâmetros. Para nosso conhecimento, apenas o modelo de dois parâmetros foi aplicado até o presente momento ao desempenho de canoagem ${ }^{(17,18)}$. Nesse sentido, o objetivo deste estudo foi comparar os parâmetros estimados a partir do modelo tradicional $\left.\right|^{(1)}$ com os estimados pelo modelo de três parâmetros ${ }^{(9)}$, e analisar a relação da VCrit estimada pelos dois modelos, com o desempenho em $6.000 \mathrm{~m}$ na canoagem, com o intuito de obter evidências de validade dos modelos de determinação da VCrit para a canoagem.

\section{MÉTODOS}

\section{Sujeitos}

Fizeram parte da amostra nove canoístas jovens (17,4 $\pm 2,4$ anos; $176,4 \pm 2,1 \mathrm{~cm} ; 68,7 \pm 7,1 \mathrm{~kg}$ ), com experiência competitiva de pelo menos um ano. Todos os sujeitos, após leitura e assinatura de um termo de consentimento informado, tanto por parte do participante quanto do responsável, no caso de menores de 18 anos, foram submetidos a duas etapas de avaliação em dias diferentes e em seqüência aleatória, sendo uma das etapas realizada para a determinação dos parâmetros do modelo de velocidade crítica e outra, para a determinação do desempenho em prova de $6.000 \mathrm{~m}$ na canoagem.

Inicialmente, essas coletas não tinham a finalidade de pesquisa, mais sim de avaliação dos atletas para obtenção de parâmetros para o treinamento.

Previamente a todos os testes havia um breve aquecimento em que os participantes percorriam $1.000 \mathrm{~m}$ em ritmo escolhido livremente. Todos os testes foram realizados em embarcação individual e oficial K-1 (5,20m de comprimento, $12 \mathrm{~kg}$ de massa). Em todas as ocasiões, foi solicitado que a última refeição prévia aos testes fosse realizada no mínimo duas horas antes dos esforços, sem controle do conteúdo e quantidade dos nutrientes ingeridos. Além disso, os atletas eram orientados a não realizar esforços vigorosos nas $24 \mathrm{~h}$ que antecediam os testes.

\section{Estimativa dos parâmetros do modelo de velocidade crítica de dois e três parâmetros em caiaque}

Foram realizados testes em quatro distâncias para predição dos parâmetros do modelo de velocidade crítica de dois (VCrit- $2_{\text {parâmetros }}$ e $\left(A_{\text {Canoagem }}{ }^{-2}\right.$ parâmetros $)$ e três parâmetros (VCrit-3 ${ }_{\text {parâmetrosı }} C_{\text {Canoagem }^{-}}$ $3_{\text {parâmetros }}$ e $V_{\text {máx }}$ ). As distâncias utilizadas foram de 200, 500, 1.000 e $1.800 \mathrm{~m}$, demarcadas em linha reta dentro de uma lagoa represada. Os testes foram realizados em dias diferentes e procurou-se fazê-los no mesmo período do dia e com as características climáticas semelhantes (preferencialmente sem vento forte a favor ou contra a trajetória das embarcações). Era solicitado que, ao sinal do avaliador, o atleta realizasse um tiro à máxima velocidade para a respectiva distância. Os atletas foram avaliados em grupos para motivá-los a tentar o melhor desempenho. O tempo foi registrado com um cronômetro manual com precisão de segundos. Para cada distância, foi calculada a velocidade média de deslocamento para posterior estimativa dos parâmetros dos modelos de dois e três parâmetros de velocidade crítica.

As equações utilizadas para estimativa dos parâmetros foram: (1) função hiperbólica com dois parâmetros, proposta por Monod e Scherrer ${ }^{(1)}$, e (2) função hiperbólica com três parâmetros, proposta por Morton $^{(9)}$. Ambas estão formuladas abaixo.

$$
\begin{aligned}
& \text { Tempo }=\frac{C A_{\text {Canoagem }}-2_{\text {parâmetros }}}{\left(\text { velocidade }- \text { V(rit- } 2_{\text {parâmetros }}\right)} \quad \text { (equação 1) } \\
& \text { Tempo }=\frac{C A_{\text {Canoagem }}-3_{\text {parâmetros }}}{\left(\text { velocidade }-V\left(\text { rit-3 } 3_{\text {parâmetros }}\right)\right.}-k \quad \text { (equação 2) } \\
& \text { onde } k=\frac{C A_{\text {Canoagem }}-3_{\text {parâmetros }}}{\left(V_{\text {máx }}-V_{\text {rit-3 }} 3_{\text {parâmetros }}\right)}, C A_{\text {Canoagem }} \text { é a capacidade }
\end{aligned}
$$

anaeróbia da canoagem, medida em metros, e $V_{\text {máx }}$ é o terceiro parâmetro fornecido pelo ajuste hiperbólico de Morton ${ }^{(9)}$ aos dados de velocidade média e tempo, e indica a velocidade máxima instantânea, teórica, a qual foi denominada originalmente por Morton ${ }^{(9)}$ como potência máxima $\left(P_{\text {máx }}\right)$.

Sobre os dados individuais de velocidade média e tempo, foram realizados ajustes não lineares, de acordo com as equações 1 e 2 . A partir desses ajustes, foram obtidos os parâmetros individuais de $\mathrm{CA}_{\text {Canoagem }}$ e VCrit para as duas equações, e de $V_{\text {máx }}$ para a equação 2. Os procedimentos adotados visavam à obtenção dos parâmetros por meio de regressões associadas ao menor somatório total dos resíduos (diferença entre o valor predito e o obtido) (Statistica ${ }^{\circ}$, versão 5.0). 


\section{Teste de 6.000 metros}

Além dos testes de predição dos parâmetros do modelo de velocidade crítica, foi realizado também um teste máximo de $6.000 \mathrm{~m}$, em dia separado dos outros testes. No entanto, para cada participante, todos os tiros foram realizados durante um período de dez dias, para que não houvesse influência do próprio treinamento nos resultados obtidos para as análises. Para o teste de $6.000 \mathrm{~m}$, os indivíduos foram avaliados dentro de uma distância de $1.000 \mathrm{~m}$, em que tiveram que realizar seis percursos completos, sem interrupção. A cada extremidade, eles tinham que dar a volta em torno da marca dos $1.000 \mathrm{~m}$. Esse procedimento foi feito individualmente, com a intenção de evitar que os atletas utilizassem a "esteira" de outra embarcação, o que facilitaria sua locomoção pela atenuação dos efeitos das forças resistivas da água ${ }^{(19)}$. O tempo foi registrado com precisão de segundos para o cálculo da velocidade média, chamada de $V_{6000 m}$. Essa velocidade foi adotada como aproximação da capacidade aeróbia.

\section{Tratamento estatístico}

Utilizou-se estatística descritiva para a apresentação dos dados (média \pm desvio-padrão). A comparação dos parâmetros VCrit- $2_{\text {pará- }}$ metros' $V$ Crit- $3_{\text {parâmetros }}$ e $V_{6000 m}$ foi feita a partir de ANOVA one way para medidas repetidas. O teste post hoc de Newman-Keuls foi utilizado para a identificação das diferenças pontuais entre as médias. Foi utilizada correlação produto-momento de Pearson entre os pares de variáveis envolvidas na estimativa de capacidade aeróbia. Entre CACanoagem $-2_{\text {parâmetros }}$ e $C A_{\text {Canoagem }}{ }^{-3}$ parâmetros' e entre os valores de $R^{2}$ foi utilizado teste $t$ para amostras pareadas. Para analisar a concordância entre VCrit- $2_{\text {parâmetros }}$ V Crit- $3_{\text {parâmetros }}$ e $V_{6000 m}$, foi aplicada a plotagem de Bland-Altman ${ }^{(20)}$, considerando as diferenças individuais entre a VCrit dos modelos de dois e três parâmetros, e a $V_{6000 m}$ (eixo y), bem como as médias obtidas entre VCrit dos modelos de dois e três parâmetros, e a $V_{6000 m}$ (eixo x). As análises foram feitas a partir do programa Statistica", versão 5.0. O nível de significância preestabelecido em todas as análises foi de $p<0,05$.

\section{RESULTADOS}

Em média, os tiros de 200, 500, 1.000 e 1.800m tiveram durações equivalentes a $46,1 \pm 2,5 \mathrm{~s}, 119,8 \pm 5,4 \mathrm{~s}, 266,0 \pm 11,6$ s e $513,9 \pm 25,6 \mathrm{~s}$, respectivamente. Já o tiro máximo de $6.000 \mathrm{~m}$ teve duração de 29,9 $\pm 1,0 \mathrm{~min}$.

A tabela 1 contém os valores individuais e as estimativas médias de VCrit- $2_{\text {parâmetros' }} V$ Crit- $3_{\text {parâmetros }} \mathrm{eV}_{6000 \mathrm{~m} \text {, }}$ mostrando que VCrit- $3_{\text {parâmetros }}$ diferiu significantemente das outras duas estimativas. A VCrit- $2_{\text {parâmetros }} \mathrm{e}$ $a V_{6000 m}$ não foram diferentes entre si $(p=0,72)$. Diferiram também entre si $(p=0,008)$ a $C A_{\text {canoagem }}-2_{\text {parâmetros }}(103,6 \pm 32,6 \mathrm{~m})$ e $C A_{\text {Canoagem }}{ }^{-3}$ parâmetros $(487,6 \pm 337,1 \mathrm{~m})$. A estimativa de $V_{\text {máx }}$ foi de 4,85 $\pm 0,46 \mathrm{~m}^{-1}$.

A figura 1, referente a um indivíduo, ilustra como a utilização do modelo de $\operatorname{Morton}^{(9)}$, com três parâmetros, afeta a localização da assíntota no eixo da potência, que demarca a VCrit, em comparação com o modelo de dois parâmetros, de Monod e Scherrer ${ }^{(1)}$. Ela mostra que a utilização do terceiro parâmetro $\left(V_{\text {máx }}\right)$ desloca substancialmente a curva para a esquerda. Ao mesmo tempo, o modelo de três parâmetros parece ajustar-se melhor aos pontos experimentais como um todo, mais especificamente àquele correspondente ao tiro de 200m. Esse melhor ajuste ao modelo de três parâmetros pode ser constatado pelo maior valor $(p<0,05)$ médio de $R^{2}$ observado $(0,991 \pm 0,010)$, em contraste com $\circ R^{2}(0,981 \pm 0,010)$ de ajuste ao modelo de dois parâmetros.
Tabela 1. Valores individuais e os correspondentes à média e desvio-padrão da VCrit- $2_{\text {parâmetros' }} V$ Crit- $3_{\text {parâmetros }} \mathrm{EV}_{6000 \mathrm{~m} \text {, }}$ erros padrão de estimativa (EPE) e os valores de $R^{2}$.

\begin{tabular}{|c|c|c|c|}
\hline Atleta & VCrit- $2_{\text {parâmetros }}\left(\mathrm{m} . \mathrm{s}^{-1}\right)$ & VCrit- $3_{\text {parâmetros }}\left(\mathrm{m} \cdot \mathrm{s}^{-1}\right)$ & $V_{6000 m}\left(m \cdot s^{-1}\right)$ \\
\hline 1 & 3,39 & 2,56 & 3,37 \\
\hline 2 & 3,29 & 3,07 & 3,26 \\
\hline 3 & 3,40 & 2,84 & 3,37 \\
\hline 4 & 3,34 & 2,63 & 3,51 \\
\hline 5 & 3,34 & 3,26 & 3,25 \\
\hline 6 & 3,03 & 2,67 & 3,28 \\
\hline 7 & 3,19 & 3,07 & 3,22 \\
\hline 8 & 3,07 & 2,63 & 3,31 \\
\hline 9 & 3,76 & 3,65 & 3,54 \\
\hline Média & $3,31 \pm 0,22^{*}$ & $2,93 \pm 0,36$ & $3,35 \pm 0,11^{*}$ \\
\hline$R^{2}$ & $0,98 \pm 0,01$ & $0,99 \pm 0,01$ & - \\
\hline EPE & $0,04 \pm 0,02$ & $0,39 \pm 0,38$ & - \\
\hline
\end{tabular}

* estatisticamente diferente de VCrit- $3_{\text {paràmetros }}(p<0,01)$

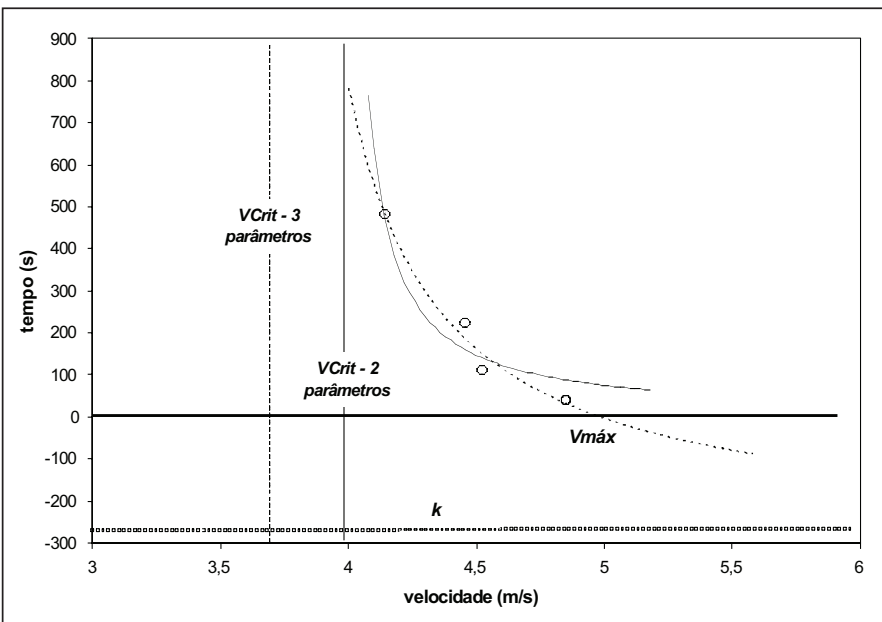

Figura 1. Ajuste dos modelos de potência crítica de dois e três parâmetros aos dados de performance na canoagem de um indivíduo (linha contínua - ajuste pela Equação 1 ; linha pontilhada - ajuste pela Equação 2; Vmáx = velocidade máxima teórica dada pelo modelo de três parâmetros)

A CA Canoagem $^{-2}{ }_{\text {parâmetros }}$ e a $C A_{\text {Canoagem }}{ }^{-3}$ parâmetros não se correlacionaram $(r=0,42 ; p=0,26)$. O mesmo ocorreu entre VCrit- $2_{\text {parâmetros }}$ e VCrit$3_{\text {parâmetros }}(r=0,65 ; p=0,06)$, embora se apresente tendência à significância, e entre VCrit-3 $3_{\text {paràmetros }} \mathrm{eV}_{6000 \mathrm{~m}}$ (figura 2). Em contraste, a correlação entre VCrit-2 parâmetros $c 0 m$ a $V_{6000 m}$ (Figura 3) foi significante.

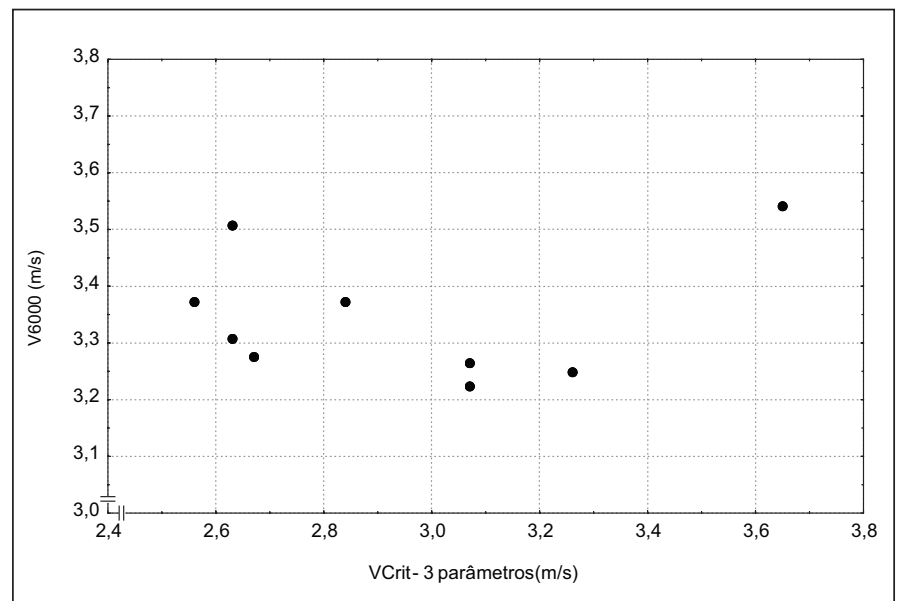

Figura 2. Relação entre a velocidade crítica obtida a partir do modelo de três parâmetros e a velocidade média mantida em $6.000 \mathrm{~m}(r=0,15 ; p>0,05)$. 


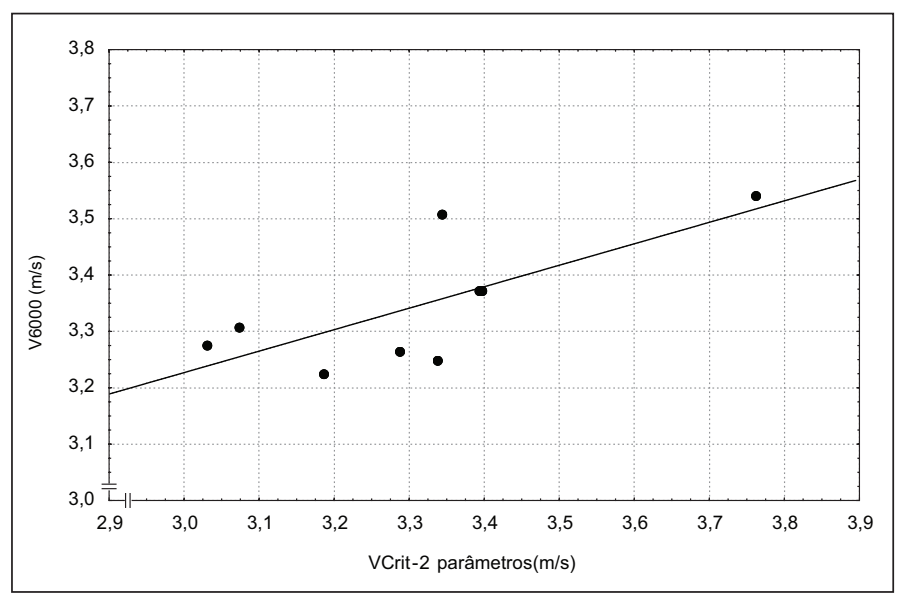

Figura 3. Relação entre a velocidade crítica obtida a partir do modelo de dois parâmetros e a velocidade média mantida em $6.000 \mathrm{~m}(r=0,72 ; p=0,03)$.

As análises de concordância pela plotagem de Bland-Altman(20) entre as estimativas de VCrit- $2_{\text {parâmetrosı }}$ VCrit- $3_{\text {parâmetros }}$ $V_{6000 m}$ são apresentadas nas figuras $4 \mathrm{~A}$ e $4 \mathrm{~B}$. As escalas no eixo y foram igualadas para facilitar as comparações visuais. Os resultados mostraram-se mais favoráveis quando confrontados os valores de VCrit-2 $2_{\text {parâmetros }}$ e $V_{6000 m}$, apresentando menores limites de concordância (IC-95\%=0,61 m/s) e diferença entre as médias $(0,03 \mathrm{~m} / \mathrm{s})$ (figura 4A). Já na comparação entre VCrit-3 parâmetros e $V_{6000 m}$ os limites de concordância foram maiores (IC$95 \%=1,43 \mathrm{~m} / \mathrm{s})$, bem como a diferença entre as médias $(0,41 \mathrm{~m} / \mathrm{s})$.

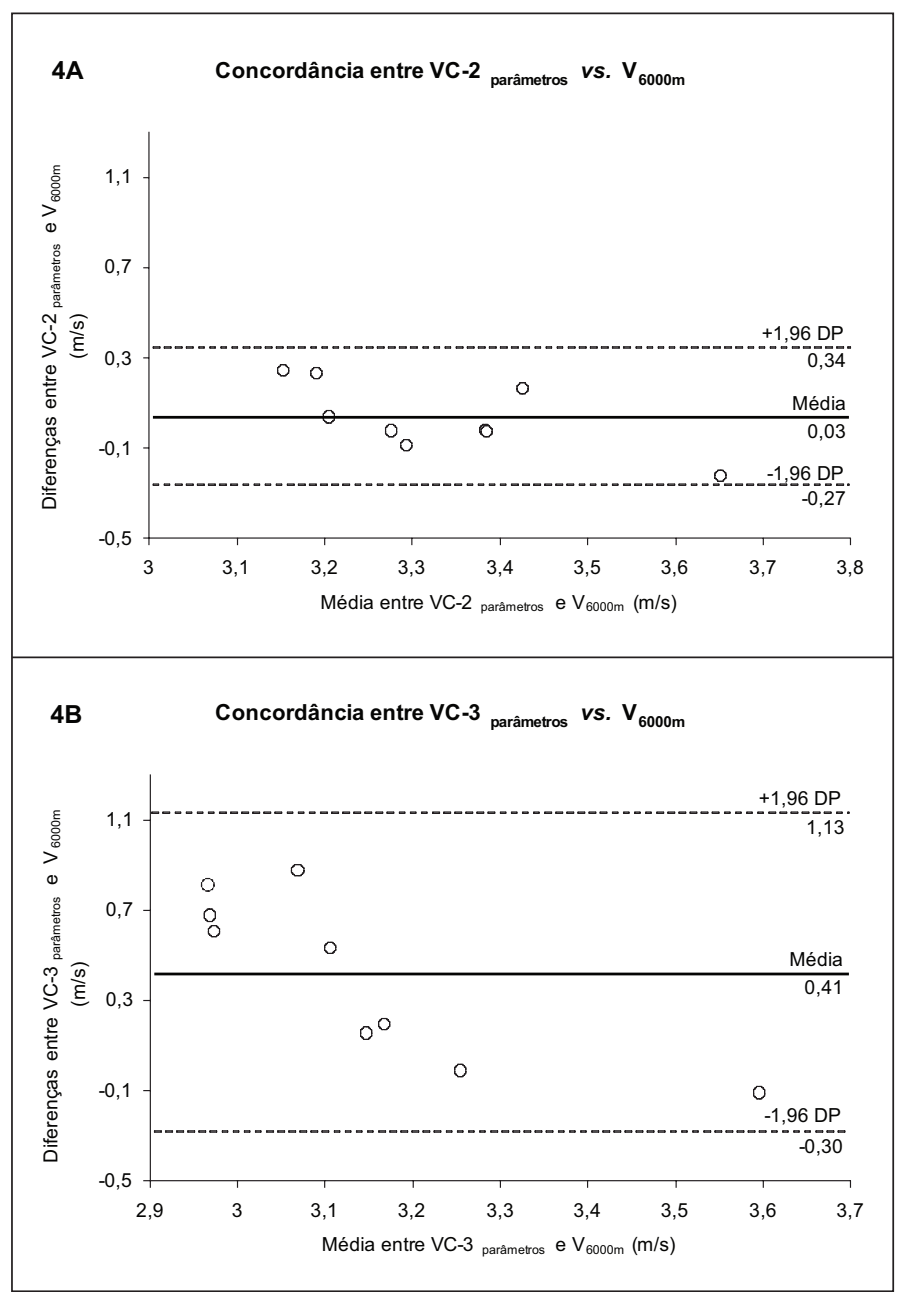

Figura 4. Análise de concordância entre as estimativas VCrit de dois parâmetros e V6000m (fig 4A) e VCrit de três parâmetros e V6000m (fig 4B).

Nota: a figura $4 \mathrm{~A}$ apresenta valores sobrepostos.

\section{DISCUSSÃO}

Para nosso conhecimento, este é o primeiro estudo a utilizar simultaneamente os modelos de velocidade crítica de dois ${ }^{(1)}$ e de três ${ }^{(9)}$ parâmetros na descrição e na interpretação da relação velocidade-tempo na canoagem. No entanto, ele pode ser comparado a trabalhos anteriores com desenhos similares, realizados com outras formas de exercício, como o remo ${ }^{(13)}$, ciclismo em ergômetro ${ }^{(10,12,21)}$ e corrida em esteira ${ }^{(11)}$.

No trabalho conduzido por Hill et al. ${ }^{(13)}$, que mais se assemelha ao nosso quanto ao tipo de locomoção, pois também acontece em fluido, compararam-se os parâmetros gerados a partir dos modelos de dois e de três parâmetros no remo, a partir de tiros realizados entre as distâncias de 200 ( 1/2 min) a 1.200m ( 3 min). Vale ressaltar que essa faixa de duração dos tiros difere substancialmente da faixa preconizada por Poole (22) para a aplicação do modelo de dois parâmetros (1-10 min). De acordo com seus resultados, a VCrit-3 parâmetros $(4,03 \pm 0,62 \mathrm{~m} / \mathrm{s})$ foi significantemente inferior a VCrit- $2_{\text {parâmetros }}\left(4,81 \pm 0,25 \mathrm{~m} \cdot \mathrm{s}^{-1}\right)$. Esse achado corrobora as diferenças reportadas no nosso estudo entre a VCrit- $3_{\text {parâmetros }}\left(2,93 \pm 0,36 \mathrm{~m} \cdot \mathrm{s}^{-1}\right)$ e a VCrit-2 parâmetros $\left(3,31 \pm 0,21 \mathrm{~m} \cdot \mathrm{s}^{-1}\right)$. Ressalta-se que, em nosso estudo, apenas a duração do teste de $200 \mathrm{~m}$ $(46,1 \pm 2,5 s)$ distanciou-se da faixa ótima proposta por Poole ${ }^{(22)}$, mas os outros três tiros foram distribuídos dentro do intervalo entre 1-10 min. Isso foi feito com o intuito de contemplar uma das condições para aplicação do modelo de três parâmetros, pois, de acordo com Morton ${ }^{(9)}$, pelo menos um teste deve aproximar-se de 1 min, ou menos. Ao confrontar o modelo de dois e três parâmetros em exercício de cicloergômetro, a PCrit- $3_{\text {parâmetros }}$ foi $8 \%$ menor em relação à $\mathrm{PCrit-} 2_{\text {parâmetros' }}$ e a CTA-3 parâmetros $_{\text {foi } 29 \% \text { maior do que CTA-2 }}$ parâmetros ${ }^{(21)}$.

A diferença entre o $R^{2}$ de ajuste dos dados experimentais nos testes de canoagem aos modelos foi significante; o modelo de três parâmetros parece ajustar-se melhor $\left(R^{2}=0,991 \pm 0,010\right)$ aos quatro pontos utilizados neste trabalho em relação ao modelo de dois parâmetros $\left(R^{2}=0,981 \pm 0,010\right)$. Os resultados de Hill et al. ${ }^{(13)}$ no remo estão de acordo com esse fato. Porém, não se pode utilizar apenas o ajuste matemático como critério de validade de um dos modelos, sobretudo porque as diferenças são pequenas. Por isso, empregou-se a performance específica em $6.000 \mathrm{~m}$, potencial discriminadora de capacidade aeróbia de canoístas, como critério de referência adicional nas análises comparativas dos modelos.

Entre os canoístas deste estudo, a correlação entre VCrit- $2_{\text {parâmetros }}$ e a $V_{6000 m}$ foi significativa, além das intensidades não diferirem. Além disso, as variáveis apresentaram boa concordância pela plotagem de Bland-Altman(20). Já a VCrit-3 ${ }_{\text {parâmetrosı }}$ além de ser significativamente menor que a $V_{6000 m}$ não apresentou correlação significante com a mesma. Portanto, apesar de o ajuste matemático aos dados experimentais ser melhor para o modelo de três parâmetros, a capacidade preditiva do desempenho aeróbio de seu parâmetro VCrit-3 parâmetros não se mostrou satisfatória.

Cabe salientar que, embora o coeficiente angular da reta representativa da relação entre $V_{C}$ rit- $2_{\text {parâmetros }}$ e $V_{6000 m}$ não ser próximo a 1 e o coeficiente linear não ser zero (figura 3), o que indica que as velocidades não são similares, percebe-se que a percentagem da diferença média entre as velocidades correspondem a 3,5 \pm 2,9\%, com percentagem máxima de 7,5\%, o que representa baixo erro na estimativa da velocidade.

Segundo Hill et al. ${ }^{(13)}$, no remo, a VCrit-2 parâmetros apresentou correlação significante e alta $(r=0,94)$ com a velocidade média mantida durante um tiro máximo de 2.000m ( 6,5 min). O mesmo não ocorreu com VCrit-3 $3_{\text {parâmetros' }}$ pois não apresentou correlação significante $(r=$ 0,38) com a performance que simulava a distância de competição. No trabalho de Hughson et al.(23), a velocidade estimada a partir do modelo 
de dois parâmetros em esteira apresentou correlação moderada ( $r$ = 0,67) com a velocidade mantida em um tiro máximo de 10.000m em pista, o qual possuía duração próxima a 30 min, assim como o tiro de $6.000 \mathrm{~m}$ entre os canoístas. O modelo de três parâmetros não foi testado por Hughson et al.(23). Todos esses resultados encontrados por outros pesquisadores reforçam nossos achados.

A PCrit- $2_{\text {parâmetrosı }}$ ou VCrit- $2_{\text {parâmetrosı }}$ parece aproximar-se do máximo estado estável de lactato e do $\mathrm{VO}_{2}(2,6,7)$, sendo associada também ao limiar de esforço percebido(24). Dessa forma, assume-se que o tempo de sustentação máxima nessa intensidade oscila em torno de 30 min $^{(4)}$, já que normalmente é esse o tempo médio de tolerância no limiar anaeróbio de membros inferiores ${ }^{(25)}$. Essa constatação empírica levou à escolha do tiro de 6.000 m como critério de performance aeróbia. No entanto, assumem-se limitações nessa opção, na medida em que há, potencialmente, variabilidade inter e intra-individual na tolerância na intensidade de limiar anaeróbio, influências da motivação na interrupção do esforço, diferenças entre padrões de recrutamento muscular em exercícios de membros superiores e inferiores, e efeitos específicos do tipo de treinamento do atleta de canoagem sobre a tolerância ao esforço aeróbio. Ainda assim, o teste fixado em distância de $6.000 \mathrm{~m}$ foi adotado neste estudo como critério de performance aeróbia, pois as limitações citadas acima são menos críticas ao se fixar a distância de teste, do que sua velocidade.

Alguns estudos têm mostrado discrepância entre o valor esperado e o realmente observado de tolerância quando da realização de exercício retangular na VCrit-2 parâmetros $^{(26,27)}$. No estudo de Pepper et al. ${ }^{(26)}$, por exemplo, o tempo de corrida mantido na VCrit- $2_{\text {parâmetros }}$ foi de apenas 16,4 \pm 6,1 min. McLellan e Cheung (27), em cicloergômetro, evidenciaram diferenças significantes entre o limiar anaeróbio individual (235 $\pm 44 \mathrm{~W})$, conhecido como IAT ${ }^{(28)}$, e a PCrit- $2_{\text {parâmetros }}(265 \pm 39 \mathrm{~W})$ em cicloergômetro. A intensidade de IAT foi mantida por 30 min com estado estável de lactato e pH sangüíneos. Na intensidade de $\mathrm{PCrit-}{ }_{\text {pa- }}$ râmetros' os indivíduos não conseguiram sustentar os 30 min de exercício, tendo ausência de estado estável de lactato. Resultado semelhante foi encontrado por Pringle e Jones ${ }^{(29)}$, onde a PCrit- $2_{\text {parâmetros }}$ foi superior à potência encontrada no máximo estável de lactato, e o tempo de tolerância na PCrit foi de 22 min sem estado estável de $\mathrm{VO}_{2}$.

Ao menos parte das diferenças reportadas entre diferentes estudos com relação ao tempo de tolerância máxima na VCrit- $2_{\text {parâmetros }}$ a a seu significado fisiológico pode estar associada a um caráter protocolo-dependente da estimativa. As variáveis intervenientes podem ser associadas à escolha das cargas utilizadas na sua identificação, com influências de diferentes amplitudes de carga no cicloergômetro ${ }^{(30)}$, e número e intensidade das cargas ${ }^{(31)}$ (emprego de intensidades maiores e/ou menores) em ergometria de membros superiores. Apesar dos achados discrepantes, a VCrit- $2_{\text {parâmetros }}$ é aceita como demarcadora entre os domínios intenso e severo de esforço(2).

De acordo com Bull et al.(12), de uma amostra composta por nove indivíduos, sete obtiveram sucesso em completar 60 min de exercício em cicloergômetro na intensidade associada a PCrit-3 ${ }_{\text {parâmetrosı }}$ finalizando com freqüência cardíaca em torno de $90 \%$ de seu valor máximo e com esforço percebido em cerca de 18 na escala de Borg ${ }^{(32)}$, sem evidenciar sinais de estabilização de ambas as variáveis. Gaesser et al. ${ }^{(10)}$ relataram que não houve diferença entre a PCrit- $3_{\text {parâmetros }}$ e a potência de ocorrência do limiar ventilatório de longo prazo ${ }^{(32)}$, a qual corresponde à intensidade em que a ventilação-minuto (VE) não sofreria elevação significativa entre 20 e 40 min de exercício. Essa intensidade, pouco empregada até o momento em outras investigações, não coincide necessariamente com o máximo estado estável de outras variáveis como lactato e $\mathrm{VO}_{2}$. Segundo Reybrouck et al. ${ }^{(33)}$, o limiar ventilatório de longo prazo (73 \pm $10 \%$ do $\mathrm{VO}_{2 \max }$ ) é superior ao limiar ventilatório de curto prazo (56 \pm $9 \%$ do $\mathrm{VO}_{2 \text { max }}$ ), tradicionalmente determinado em teste progressivo, por meio do aumento da (VE) ou da relação $V E N \mathrm{NO}_{2}$, sem aumento concomitante de $\mathrm{VEN} \mathrm{NCO}_{2}{ }^{(34)}$. Apesar de a concentração de lactato no sangue e de o $\mathrm{VO}_{2}$ não terem aumentado entre os minutos 20 e 40 do teste na intensidade do limiar ventilatório de longo prazo, a 20W acima dessa intensidade o lactato apresentou elevação, não sendo acompanhado pelo aumento significante do $\mathrm{VO}_{2}$. Dessa forma, o significado fisiológico

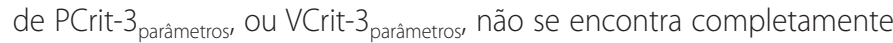
elucidado. No entanto, os diversos estudos sugerem que o tempo de tolerância na VCrit- $3_{\text {parâmetros }}$ é maior que em VCrit- $2_{\text {parâmetros }}$.

Em esteira rolante, Housh et al. ${ }^{(11)}$ compararam as intensidades relativas de ocorrência da VCrit- $2_{\text {parâmetros }}$ e VCrit- $3_{\text {parâmetros }}$ em termos de respostas fisiológicas obtidas em teste progressivo com estágios de 3 min. Novamente, encontraram-se cargas diferentes referentes aos valores de VCrit. A VCrit- $2_{\text {parâmetros }}$ ocorreu a $94 \%$ do VO 2maxı $_{1} 96 \%$ da freqüência cardíaca máxima e à concentração de lactato plasmático igual a $71 \%$ do valor de pico na exaustão. Já VCrit-3 parâmetros ocorreu a $89 \%$ do $\mathrm{VO}_{2 \text { maxı }} 93 \%$ da freqüência cardíaca máxima e à concentração de lactato plasmático de 63\% do valor de pico. Esses dados sugerem que ambos superestimaram a faixa normalmente aceita como transição entre os domínios intenso e severo de esforço(2). Nesse estudo, a duração dos testes ficou entre 2 e12 min. Sendo assim, não houve um ponto preconizado por Morton ${ }^{(9)}$ com duração igual ou inferior a 1 min.

Não foi objetivo deste estudo comparar $\mathrm{CA}_{\text {canoagem }} 2_{\text {parâmetros }}$ e CACanoagem ${ }^{-3}$ parâmetros' uma vez que suas estimativas não são equivalentes, porque partem de pressupostos teóricos diferentes. Mesmo assim, esses construtos merecem algumas considerações. Entendemos que a $\mathrm{CA}_{\text {cano- }}$ agem -2 parâmetros não representa toda a energia potencial anaeróbia contida nos músculos ativos, já que para isso seria necessária a depleção de toda a quantidade de glicogênio disponível para o trabalho. Ela parece constituir apenas uma parcela depletada até que processos periféricos ${ }^{(35)}$, ou centrais ${ }^{(24)}$, associados à acidose metabólica e/ou esgotamento de fosfagênios, por exemplo, conduzissem a processos de fadiga, resultando em diminuição significativa do desempenho anaeróbio, ou até mesmo na deterioração da atividade contrátil, a despeito da não utilização integral dos estoques de glicogênio. Dessa forma, o valor de $\mathrm{CA}_{\text {canoagem }}{ }^{-}{ }_{\text {parâmetros }}$ de 103,6 \pm 32,6m parece razoável. De acordo com a formulação de três parâmetros de Morton, após atividades severas exaustivas haveria reserva anaeróbia potencial remanescente por conta dos estoques de glicogênio preservados ${ }^{(10)}$. Assim, o valor de $\mathrm{CA}_{\text {Canoagem }}{ }^{-3}$ parâmetros $d e$ 487,6 土 337,1 m também parece razoável, porque ela incluiria a "reserva" anaeróbia (de glicogênio) remanescente após exercício severo exaustivo. Assumindo que a glicogenólise anaeróbia gera três mols de ATP por mol de glicogênio e que a fosforilação oxidativa a partir do mesmo substrato gera em torno de 36 mols de ATP, chega-se à conclusão de que o metabolismo anaeróbio apresenta eficiência energética de cerca de $8,3 \%$ do metabolismo aeróbio. Dessa forma, se os estoques de glicogênio que resultam em 487,6 \pm 337,1 m fossem utilizados aerobiamente, a distância possível de ser vencida seria de aproximadamente $5.900 \mathrm{~m}$. Esse valor aproxima-se da distância de 6.000m, percorrida em intensidade equivalente a VCrit- $2_{\text {parâmetros }}$ utilizada neste estudo como aproximação indireta de capacidade aeróbia. Mesmo que os valores

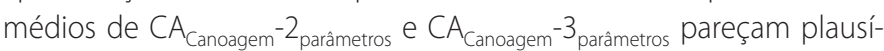
veis, sua acuidade e validade têm sido questionadas ${ }^{(17,36)}$ e merecem tratamento experimental em outras investigações.

De forma geral, nossos resultados apontam que a utilização de dois diferentes modelos matemáticos de velocidade crítica na canoagem interfere em sua capacidade de predição do desempenho aeróbio. Indica-se que, na prática do treinamento, haja preferência para a estimativa de VCrit- $2_{\text {parâmetros }}$ em vez de VCrit- $3_{\text {parâmetros' }}$ sobretudo quando o objetivo for avaliar atletas e prescrever seus treinamentos com base 
nesse parâmetro. A abordagem de dois parâmetros permitiria melhor aproximação da $V_{6000 m}$ ou da velocidade mantida por cerca de 30 min. Se os pressupostos do estudo estiverem corretos, a VCrit- $2_{\text {parâmetros }}$ pode ser utilizada na escolha de cargas de treinamentos, pois em intensidades iguais ou menores que ela, os estímulos seriam característicos do domínio intenso ${ }^{(18)}$, ao passo que acima dela os esforços seriam severos. As adaptações de longo prazo dependem, em grande medida, da aproximação dessas intensidades por meio de testes. Aparentemente, a VCrit-3 parâmetros subestima a intensidade de transição metabólica (máximo estado estável de lactato), o que não favoreceria a escolha das cargas de treinamento com a mesma acuidade.

Com isso, apesar de se constituir, do ponto de vista lógico e matemático, como alternativa de aplicação do modelo de velocidade crítica, o modelo de três parâmetros com a inserção da $\bigvee_{\text {máx }}$ não agrega valor adicional à predição do desempenho aeróbio na canoagem. Assim, o papel prático na avaliação e predição do desempenho dos parâmetros do modelo de Morton ${ }^{(9)}$ não parece estar ainda esclarecido, sobretudo em virtude de achados recentes, críticos com relação à aplicação do modelo e com relação ao significado fisiológico dos seus parâmetros ${ }^{(37)}$.

Dentre as limitações do presente estudo, destaca-se a ausência de medidas fisiológicas que poderiam garantir a observação de estado estável de variáveis como o lactato sangüíneo e $\mathrm{VO}_{2}$ durante o tiro máximo de $6.000 \mathrm{~m}$. Ressalta-se, entretanto, que o registro dessas variáveis seria tecnicamente problemático. A coleta de lactato no sangue, em particular, exigiria a interrupção do esforço, o que impediria a tomada do tempo de melhor desempenho contínuo na distância prefixada. Outra limitação a ser considerada é a interferência do baixo número de sujeitos avaliados sobre a determinação da relação entre as variáveis. Essa interferência pode implicar mudanças na força da relação entre variáveis, ou até mesmo na significância dessa correlação, assim como interferir nos parâmetros da função preditiva, representativa dessa relação. De fato, no presente estudo, foi observado que o coeficiente angular da reta representativa da relação entre $V$ Crit- $2_{\text {paràmetros }}$ e $V_{6000 m}$ não é próximo a 1 e o coeficiente linear não é zero (figura 3), o que indica que as velocidades não são similares. No entanto, percebe-se que a percentagem da diferença média entre as velocidades corresponde a 3,5 $\pm 2,9 \%$, com percentagem máxima de $7,5 \%$, o que representa baixo erro na estimativa da velocidade.

\section{CONCLUSÕES}

Os resultados deste estudo sugerem que o modelo de velocidade crítica de dois parâmetros fornece o valor de VCrit mais adequado para a avaliação aeróbia de canoístas, uma vez que a VCrit- $2_{\text {parâmetros }}$ e $V_{6000 m}$ não diferiram, além de ser altamente correlacionadas. A VCrit-3 parâmetros subestima a velocidade que pode ser mantida por aproximadamente 30 min, não se relacionando estatisticamente com a mesma. A VCrit3 parâmetros apresenta, portanto, baixa capacidade preditiva de desempenho aeróbio. Sendo assim, este estudo acrescentou elementos de validade ao modelo original de velocidade crítica de dois parâmetros proposto por Monod e Scherrer ${ }^{(1)}$. Dessa forma, sugere-se que, em termos práticos, a VCrit-2 ${ }_{\text {parâmetros }}$ seja utilizada em detrimento da VCrit$3_{\text {parâmetros }}$ quando se objetiva avaliar canoístas e promover estratégias de treinamento com base em parâmetros de intensidade.

Todos os autores declararam não haver qualquer potencial conflito de interesses referente a este artigo.

\section{REFERÊNCIAS BIBLIOGRÁFICAS}

1. Monod H, Scherrer J. The work capacity of a synergic muscular group. Ergonomics 1965;8:329-38.

2. Gaesser GA, Poole D. The slow component of oxygen uptake kinetics in humans. Exerc Sport Sci Rev 1996;24:35-70

3. Moritani T, Nagata A, DeVries HA, Muro M. Critical power as a measure of physical work capacity and anaerobic threshold. Ergonomics 1981;24:339-50.

4. Brickley G, Doust J, Williams CA. Physiological responses during exercise to exhaustion at critical power. Eur J Appl Physiol 2002;88:146-51.

5. Poole DC, Ward SA, Gardner GW, Whipp BJ. Metabolic and respiratory profile of the upper limit for prolonged exercise in man. Ergonomics 1988;31:1265-79.

6. Poole DC, Ward SA, Whipp BJ. The effects of training on the metabolic and respiratory profile of high-intensity cycle ergometer exercise. Eur J Appl Physiol 1990;59:421-9.

7. Hill DW, Smith JC. Determination of critical power by pulmonary gas exchange. Can J Appl Physiol 1999;24:74-86.

8. Hill DW, Smith JC. A method to ensure the accuracy of estimates of anaerobic capacity derived using the critical power concept. J Sports Med Phys Fitness 1994;34:23-37.

9. Morton RH. A 3-parameter critical power model. Ergonomics 1996;39:611-9.

10. Gaesser GA, Carnevale TJ, Garfinkel A, Walter DO, Womack CJ. Estimation of critical power with nonlinear and linear models. Medicine and Sci Sports Exerc 1995;27:1430-8.

11. Housh TJ, Cramer JT, Bull AJ, Johnson GO, Housh DJ. The effect of mathematical modeling on critical velocity. Eur J Appl Physiol 2001;84:469-75.

12. Bull AJ, Housh TJ, Johnson GO, Perry SR. Effect of mathematical modeling on the estimation of critical power. Med Sci Sports Exerc 2000;32:526-30.

13. Hill DW, Alain C, Kennedy MD. Modeling the relationship between velocity and time do fatigue in rowing. Med Sci Sports Exerc 2003;35:2098-105.

14. Vandewalle H, Kapitaniak B, Grün S, Raveneau S, Monod H. Comparison between a 30-s all-out test and time-work test on a cycle ergometer. Eur J Appl Physiol 1989;58:375-81

15. Hill DW, Ferguson CS. A physiological description of critical velocity. Eur J Appl Physiol 1999;79:290-3.

16. Wakayoshi K, Ikuta K, Yoshida T, Udo M, Moritani T, Mutoh Y, et al. Determination and validity of critical velocity as an index of swimming performance in the competitive swimmer. Eur J Appl Physiol Occup Physiol 1992;64:153-7.

17. Nakamura FY, Borges TO, Brunetto AF, Franchini E. Correlação entre os parâmetros do modelo de potência crítica no cicloergômetro de membros superiores e no caiaque. Rev Bras Ci Mov 2005;13:41-8

18. Fontes EB, Nakamura FY, Gobbo LA, Altimari LR, Melo JC, Carvalho FO, et al. Does critical velocity represent maximal steady state lactate in canoe/kayak flatwater? The FIEP Bulletin 2005;75:427-30.

19. Ribeiro LFP, Galdino R, Balikian P. Resposta lactacidêmica de nadadores e triatletas em função da utilização de "esteira" durante natação em velocidade correspondente ao limiar anaeróbio. Rev Paul Educ Fís 2001;55-62.

20. Bland JM, Altman DG. Statistical methods for assessing agreement between two methods of clinical measurement. The Lancet 1986:1(8486);307-10.

21. Hill DW. The relationship between power and time to fatigue in cycle ergometer exercise. Int J Sports Med 2004;25:357-61.

22. Poole DC. Letter to the editor-in-chief. Med Sci Sports Exerc 1986;26:1335-40.

23. Hughson RL, Orok CJ, Staudt LE. A high velocity treadmill running test to assess endurance running potential. Int J Sports Med 1984;5:23-5.

24. Nakamura FY, Gancedo, MR, Albuquerque da Silva L, Lima JRP, Kokubun E. Utilização do esforço percebido na determinação da velocidade crítica em corrida aquática. Rev Bras Med Esporte 2005;11:1-5.

25. Tegtbur U, Busse MW, Braumann KM. Estimation of an individual equilibrium between lactate production and catabolism during exercise. Med Sci Sports Exerc 1993;25:620-7.

26. Pepper ML, Housh TJ, Johnson GO. The accuracy of the critical velocity test for predicting time to exhaustion during treadmill running. Int J Sports Med 1992;13:121-4.

27. McLellan TM, Cheung KSY. A comparative evaluation of the individual anaerobic threshold and the critical power. Med Sci Sports Exerc 1992;24:543-50.

28. Stegmann H, Kinderman W, Schnabel A. Lactate kinetics and individual anaerobic threshold. Int J Sports Med 1981;2:160-5.

29. Pringle JSM, Jones AM. Maximal lactate stedy state, critical power and EMG during cycling. Eur J Appl Physiol 2002;88:214-26.

30. Housh DJ, Housh TJ, Bauge SM. A methodological consideration for the determination of critical power and anaerobic work capacity. Res Q Exerc Sport 1990;61:406-9.

31. Calis JFF, Denadai BS. Influência das cargas selecionadas na determinação da potência crítica determinada no ergômetro de braço em dois modelos lineares. Rev Bras Med Esporte 2000;6:1-4.

32. Borg GAV. Psychophysical bases of perceived exertion. Med Sci Sports Exerc 1982;14:377-81.

33. Reybrouck T, Ghesquiere J, Cattaert A, Fagard R, Amery A. Ventilatory thresholds during short- and long-term exercise. J Appl Physiol 1983;55:1694-700.

34. Wasserman K, Whipp B, Koyal NS, Beaver WL. Anaerobic threshold and respiratory gas exchange during exercise. J Appl Physiol 1973;35:236-43.

35. Fabiato A, Fabiato F. Effects of pH on the myofilaments and sarcoplasmic reticulum of skinned cells from cardiac and skeletal muscle. J Physiol 1978;276:233-55.

36. Papoti M, Zagatto AM, Freitas Júnior PB, Cunha SA, Martins LEB, Gobatto CA. Utilização do intercepto-y na avaliação da aptidão anaeróbia e predição da performance de nadadores treinados. Rev Bras Med Esporte 2005;11:126-30

37. Chatagnon M, Pouilly J-P, Thomas V, Busso T. Comparison between maximal power in the powerendurance relationship and maximal instantaneous power. Eur J Appl Physiol 2005;94:711-7. 